www.jmscr.igmpublication.org

Index Copernicus Value: 79.54

ISSN (e)-2347-176x ISSN (p) 2455-0450

crossref DOI: https://dx.doi.org/10.18535/jmscr/v7i5.27

Journal Of Medical Science And Clinical Research

IGM Publication

An Official Publication of IGM Publication

\title{
Does Secretor Status of ABO Blood Group in Saliva Influence the Risk of Hypertension and Urinary Tract Infection in Diabetic Patients?
}

Authors

Shanmugapriya Thiagarajan ${ }^{1}$, Selvaraj Stephen ${ }^{2}$, Sarangapani Kanagamuthu ${ }^{3}$, Stanley Ambrose $^{4}$, Pragasam Viswanathan ${ }^{5}$, Palanivel Chinnakali ${ }^{6}$, Rajesh Nachiappa Ganesh ${ }^{7}$

${ }^{1}$ Department of Microbiology, Indira Gandhi Government General Hospital and Postgraduate Institute, Puducherry - 605001

${ }^{2}$ Department of Microbiology, Mahatma Gandhi Medical College and Research Institute, Puducherry, India- 607403

${ }^{3}$ Department of Microbiology, Indira Gandhi Government General Hospital and Postgraduate Institute, Puducherry, India- 605001

${ }^{4}$ Department of Medicine Indira Gandhi Government General Hospital and Postgraduate Institute, Puducherry, India - 605001

${ }^{5}$ Vellore Institute of Technology, Vellore, India

${ }^{6}$ Department of Preventive and Social Medicine, Jawaharlal Institute of Postgraduate Medical Education and Research, Puducherry India - 605006

${ }^{7}$ Department of Pathology, Jawaharlal Institute of Postgraduate Medical Education and Research, Puducherry, India - 605006

*Corresponding Author

Dr Rajesh Nachiappa Ganesh

Additional Professor, Department of Pathology, JIPMER, Puducherry - 605006, India

Email: drngrajesh@gmail.com, Mobile-91-9791976021

\begin{abstract}
Secretor status of blood group antigens in saliva is implicated to be associated with several infectious and immune related diseases. Prospective analytical study carried out in a tertiary hospital in South India to study the association of ABO secretor status with type II diabetes mellitus and its microangiopathic complications. A total of 198 patients with type II diabetes mellitus, diagnosed and treated in the hospital were studied. 200 healthy controls were studied from healthy volunteers working in the hospital. ANOVA, Pearson's Chi Square Test and Cox Univariate and Multivariate analysis are the statistical methods used to analyse the data. $21.2 \%$ of diabetics and $33.5 \%$ of healthy controls were ABO secretors. Secretors were found to have a statistically significant risk of association with type II diabetes mellitus when adjusted for other potential confounding factors. Secretor status was in addition found to have significant association with hypertension. Non-secretors were significantly associated with increasing age of the patient and had increased risk of urinary tract infections (UTI). The risk of microangiopathic complications due to type II diabetes mellitus were significantly higher with increasing levels of glycated haemoglobin (HbAlc). In our study, we found significant association of secretors with type II diabetes mellitus and hypertension while non-secretors were associated with increased risk of urinary tract infections.
\end{abstract}

Keywords: Secretor status, Type II diabetes mellitus, Hypertension, UTI, microangiopathic Complications. 


\section{Introduction}

A secretor refers to a patient who secretes blood group antigens in fluids such as saliva, sweat, tears, semen, and serum. $\mathrm{ABH}$ refers to ' $\mathrm{A}$ ' and ' $\mathrm{B}$ ' antigens of $\mathrm{ABO}$ blood group and ' $\mathrm{H}$ ' the heterogenic constituent of all $\mathrm{ABO}$ types including type ' $\mathrm{O}$ '. $\mathrm{H}$ antigen is the indirect gene product expressed as fucose-containing glycan unit and it resides on glycoproteins or glycolipids of erythrocyte membranes or on mucin glycoproteins in secretions. $\mathrm{H}$ antigen is expressed by two genes FUT1(H) gene predominantly in erythroid tissues; FUT2 (Secretor) gene is expressed predominantly in secretory tissues ${ }^{1}$. Among all body fluids, saliva is the rich source to determine Secretor status ${ }^{2}$.

Saliva has protein, glycoprotein, peptide and carbohydrate constituents in addition to inorganic substances. Salivary glycoproteins are in constant interaction with variety of bacteria in the oral flora through their carbohydrate moiety. Fucosyltransferase 2 (FUT2) is responsible for fucosylation of glycoforms and this influences the scaffolding of Leb/y and blood type motifs. These glycans act as receptors mediating the adhesion of several bacteria in oral cavity, few examples including sT-Ag for Streptococcal strains and Le determinants for H. pylori strains. Fucosylation of gPRP disaccharide blocks bacterial binding. Nonsecretors have inactive form of FUT2 genotype and phenotype, thereby influencing salivary glycoproteins with significantly lower levels of fucosylation of the released N-glycans.

Blood group and secretor status have significant influence in inter-individual variation in salivary glycosylation and consequently on interaction with bacterial milieu in the oral cavity ${ }^{3}$.

The ability or inability to secrete $\mathrm{ABH}$ blood group substances in body fluid has been studied with susceptibility to a number of pathological conditions. Previous studies have indicated that non-secretors are more prone to certain diseases such as autoimmune diseases like insulindependent diabetes mellitus ${ }^{4}$, ankylosing spondylitis, reactive arthritis, psoriatic arthropathy, Sjogren's syndrome, multiple sclerosis $^{5}$, peptic ulcers ${ }^{6}$, vaginal candidiasis ${ }^{7}$, etc. Non-secretors have increased inflammatory response (increased C-reactive protein, erythrocyte sedimentation rate and the body temperature) to urinary tract infections (UTI) than Secretors ${ }^{8}$.

Efforts attempting to supplement blood group substances prepared from edible food are proposed for prevention and treatment of various bacterial and viral strains but however at the moment there is little scientific evidence on the same with conflicting opinions ${ }^{9}$.

FUT2 polymorphisms and secretor status is also proven to be one of the key drivers affecting the individual variations in gut microbiota. Association of non-secretor phenotype with various diseases have been implicated in the increased risk for Crohn's disease, type I diabetes, urinary tract infections, candidiasis and viral infections such as rota virus and norovirus infections in the $\mathrm{GIT}^{10,}{ }^{11}$. The influence of secretor status on antibiotic treatment of enteric pathogens such as Salmonella and Clostridium difficile is also reported ${ }^{12}$.

Type II Diabetes mellitus (DM) is a metabolic disease which has a genetic predisposition, although environmental factors do play a significant role in its genetic expression. Like many other inherited traits, $\mathrm{ABH}$ secretor status is also genetically pre-determined and therefore we studied an association with diabetes mellitus and its complications.

\section{Subjects and Methods}

The study design was prospective analytical and the study group was recruited from the people who came for medical and surgical check-up in the outpatient department. Patients already diagnosed to have non-insulin dependent diabetes mellitus (NIDDM) were taken as study population (type II DM). Healthy individuals who are not diabetic (non-DM) were taken as healthy control from volunteers among health care workers in the hospital. Both males and females of age ranging 
from 18 to 74 years were recruited. A total of 198patients who had type II diabetes mellitus samples were subjected to secretor status identification from saliva samples. 200 healthy controls were recruited in the study and their secretor status from saliva was also determined. Ocular examination was performed and retinopathy was graded according to the modified Airlie House classification system. The moderate/severe diabetic retinopathy (DR) was defined as case: grade $>=30$; control: grade $<14$. Physical findings as assessed by Physician were also documented in the study group. Patients who presented with symptoms of urinary tract infections were studied for routine urine examination with urine microscopy and culture and sensitivity. Majority of the patients in the study group (patients with type II diabetes mellitus) were not aware of the exact onset of the disease. Volunteers in the control group were also screened for blood pressure, urine albumin, sugar and microscopy and serum creatinine. Only those who were diagnosed in the hospital with records were included to avoid confounding factors. Ethical clearance was obtained from Institutional ethical committee. Chi-square test was performed to assess the statistical significance between the two variables.

\section{Sample collection and processing}

Collection of saliva was performed after thoroughly rinsing the mouth with water. All the saliva samples were collected 2-3 hours after the usual breakfast time. About $2 \mathrm{ml}$ of saliva was collected in a dry sterile tube. Saliva tube was kept for 20 minutes in a boiling water bath at $100^{\circ} \mathrm{C}$ to denature the salivary enzymes. It was then cooled to room temperature and centrifuged for 5 minutes at $1000 \mathrm{~g}$, supernatant was collected. Secretor status of the saliva samples were identified by using adsorption inhibition technique ${ }^{13}$. The procedure is briefly mentioned below.

Six test tubes were labelled C, 2, 4, 8, 16, 32. C refers to the control tube, to which no saliva was added. $50 \mu$ of saliva was added into the second tube labelled 2. For the rest of the tubes labelled 4,
8,16 and 32 , saliva was titrated by doubling dilution using normal saline. $50 \mu \mathrm{l}$ of anti A serum was added to each test tube. All the tubes were shaken well and left undisturbed for 10-15 minutes. One drop of a suspension of group A RBCs in a concentration of 5\% was added to each tube. The tubes were left to stand for 5 minutes and subsequently centrifuged at $3500 \mathrm{rpm}$ for 20 seconds. The sediment was decanted on a slide and studied for presence of agglutination. (Fig 1) The degree of agglutination was graded as follows,

Grade 0 - all red blood cells are discrete and evenly distributed in surrounding fluid (no agglutination)

Grade 1 - Few or very occasional clumps

Grade 2 - moderate aggregates

Grade 3 - composed of moderate to large aggregates

Grade 4- very large aggregates, very few cells remain free without agglutination.

Control tubes always showed grade 4 Agglutination. The procedure was repeated with group B RBC and anti B serum and subsequently with group $\mathrm{O}$ RBCs and anti $\mathrm{H}$ serum. This is based on the principle that when saliva has blood group antigens, the antibodies in the serum of appropriate dilution will be utilized and will result in a negative agglutination when the corresponding blood group RBC's are added. Grades $0,1,2,3$ agglutination are interpreted as secretor while Grade 4 agglutination is interpreted as non-secretor.

$2 \mathrm{ml}$ of blood was collected in ethylene diamine tetra acetic acid (EDTA) coated vaccutainer tubes and $\mathrm{ABO}$ blood group and $\mathrm{Rh}$ typing was carried out by forward and reverse grouping techniques using test tube method as per standard operating procedure in the Institute. $2 \mathrm{ml}$ of serum was collected from all the controls once and $2 \mathrm{ml}$ serum was collected from all the patients in both fasting and post-prandial state to assess the blood sugar levels (by Glucose oxidase-perioxidase method), serum creatinine by (Modified Jaffe's method), HbA1c (by Immuno-turbidometric method) and 
serum albumin (Bromocresol green dye binding method). Biochemical measurements were performed on fully authomated analyzer (Beckman Coulter) using colorimetric method. Urine examination was carried out for assessment of albuminuria (by Immuno-turbidometric method) and presence of sugar in urine by using strip test and subsequently urine was centrifuged and studied for deposits and casts by light microscopic examination. Each test was done in duplicate to ensure precision and accuracy and only concordant results were included for the analysis. 20 patients in the study population and 16 control samples had discordant results for secretor status assessment, which were excluded from the study. Duration of diabetes mellitus in the study population ranged from newly diagnosed patients to patients who had diabetes for more than 30 years.

Clean catch mid stream urine is collected in a sterile wide mouthed container of capacity $50 \mathrm{ml}$. Urine samples were inoculated in MacConkey agar and Blood agar using a sterile $4 \mathrm{~mm}$ platinum wired calibrated loop and incubated overnight at $37^{\circ} \mathrm{C}$ for 24 hours. The specimen was considered significant bacteriuria when the growth was $>10^{5}$ colony forming unit (CFU/ml). The isolates were identified till species level using standard biochemical tests ${ }^{14}$.Antibiotic sensitivity testing was done following the Kirby- Bauer disc diffusion method in Muller Hinton agar as per Clinical and Laboratory Standards Institute (CLSI) guidelines ${ }^{15}$.

\section{Results}

A total of 398 samples were processed, 198 $(49.75 \%)$ of whom were from patients with type II diabetes mellitus and $200(51.25 \%)$ were from normal healthy controls. Among the patients in the study group, $42(21.2 \%)$ were secretors while in the control population, 67 (33.5\%) were secretors. (Table 1)

Table 1 Secretor status in the study group and Control population

\begin{tabular}{|l|c|c|c|}
\hline S.No & $\begin{array}{c}\text { Secretor status } \\
\text { in study } \\
\text { subjects n (\%) }\end{array}$ & $\begin{array}{c}\text { Secretor status } \\
\text { in healthy } \\
\text { controls n (\%) }\end{array}$ & $\begin{array}{c}\text { p value } \\
\text { (Chi square } \\
\text { test) }\end{array}$ \\
\hline 1 & $42(21 \%)$ & $67(33.5 \%)$ & 0.008 \\
\hline
\end{tabular}

The detailed split of the secretor status among various blood groups reflected that secretors were highest among $\mathrm{O}$ blood group followed by patients with A group. The picture was similar among study subjects and healthy control in the distribution of blood groups and secretors. However, non-secretors were found to be more common among study population in groups $\mathrm{O}, \mathrm{A}$ and $\mathrm{AB}$ as highlighted in the table. (Table 2)

Table 2 Distribution of Secretor status among Blood groups and study population

\begin{tabular}{|l|c|c|c|c|c|c|}
\hline Blood & \multicolumn{3}{|c|}{ Study Subjects } & \multicolumn{3}{c|}{ Healthy Controls } \\
\hline & Total & Secretor & Non secretor & Total & Secretor & Non secretor \\
& $198(\%)$ & $42(21 \%)$ & $156(78 \%)$ & $200(\%)$ & $67(33.5 \%)$ & $133(66.5 \%)$ \\
\hline O & $72(36)$ & $18(25)$ & $54(75)$ & $76(38)$ & $33(43.4)$ & $43(56.6)$ \\
\hline A & $56(28)$ & $15(26.8)$ & $41(73.2)$ & $64(32)$ & $24(37.5)$ & $40(62.5)$ \\
\hline B & $58(29)$ & $08(13.8)$ & $50(86.2)$ & $50(25)$ & $08(16)$ & $42(84)$ \\
\hline AB & $12(6)$ & $01(8.3)$ & $11(91.7)$ & $10(5)$ & $02(20)$ & $08(80)$ \\
\hline
\end{tabular}

A total of 52 patients presented with symptoms of lower urinary tract infections (UTI) in the study population. Among them, 47 (90.4\%) patients had white blood cell (WBC) casts in urine microscopy and $40(76.9 \%)$ of them had positive urine culture. In patients with symptoms of UTI, 44 patients $(84.6 \%)$ were non-secretors. (Table 3)
Table 3 Organisms identified in Culture of Urinary Tract Infection patients in the Study group

\begin{tabular}{|l|c|c|}
\hline S.No & Organism Total $\mathrm{n}=40$ & Number/Percentage \\
\hline 1 & Escherichia coli & $28(70 \%)$ \\
\hline 2 & Klebsiella pneumoniae & $8(20 \%)$ \\
\hline 3 & Pseudomonas aeruginosa & $2(5 \%)$ \\
\hline 4 & Enterococcus fecalis & $1(2.5 \%)$ \\
\hline 5 & Proteus mirabilis & $1(2.5 \%)$ \\
\hline
\end{tabular}

Page 156 
Patients with diabetes mellitus were assessed for the duration of the disease both as continuous variables and as categorical variables. Duration of diabetes was categorized as patients who had the disease for $<3$ years, 3-6, 6-9, 9-12 and >12 years and the number of patients in each category was $63,65,22,18$ and 30 respectively. Statistical analysis was performed to correlate the duration with various complications, age, sex, hypertension and secretor status.

Univariate analysis was performed for all the parameters. Age of the patient is significantly higher in the study (diabetes) group when compared with healthy control group. Mean age of the patients in control group is 39.8 and in the diabetes group, it is 46.63. Using Two sample ttest, the $\mathrm{p}$ value is $<0.001$. Hypertension is significantly associated with study group ( $\mathrm{p}$ value is $<0.001$; Pearson's Chi square test). Nonsecretors were significantly more common in study group (diabetes) when compared with control group while secretors were significantly less common in the study group ( $p$ value is $<0.001$; Pearson's Chi square test). Rest of the parameters were not significantly associated in Univariate analysis between the two groups. (Tables 4,5,6).

Table 4 Age - Controls vs type II Diabetes group (Two sample t test with unequal variances)

\begin{tabular}{|l|c|c|c|c|c|c|}
\hline Group & Observations & Mean & Std. Err & Std. Dev & \multicolumn{2}{c|}{$95 \%$ conf. interval } \\
\hline 0 & 200 & 39.805 & 0.5273284 & 7.45755 & 38.76513 & 30.84487 \\
\hline 1 & 198 & 46.63131 & 0.5972146 & 8.403554 & 45.45356 & 47.80907 \\
\hline Combined & 398 & 43.20101 & 0.432981 & 8.637088 & 42.34987 & 44.05214 \\
\hline Diff & -6.826313 & 0.796706 & & -8.392695 & -5.259931 \\
\hline Diff $=$ mean $(0)$ - mean (1) & Satterthwaite's degree of freedom $=389.531$ \\
Ho:diff $<0$ & Ha: diff $=0$ \\
Ha: diff $<0$ & Ha: diff $>0$ \\
Pr $(\mathrm{T}<\mathrm{t})=0.00000$ & $\operatorname{Pr}(\mathrm{T}>\mathrm{t})=0.00000$ & $\operatorname{Pr}(\mathrm{T}>\mathrm{t})=1.0000$ \\
\hline
\end{tabular}

Table 5 Hypertension - Controls vs type II Diabetes group (group $0=$ healthy control, $1=$ diabetes mellitus; HT $0=$ hypertension absent, $1=$ htn present)

\begin{tabular}{|l|c|c|c|}
\hline HT & Group 0 & Group 1 & Total \\
\hline 0 & 193 & 64 & 257 \\
& 96.50 & 32.32 & 64.57 \\
\hline 1 & 7 & 134 & 141 \\
& 3.50 & 67.68 & 35.43 \\
\hline Total & 200 & 198 & 398 \\
\multicolumn{2}{|l|}{ Pearson Chi square $(1)=179.1355$} & 100.00 \\
\hline
\end{tabular}

Table 6 Secretor status in Controls and type II DM patients (Group $0=$ healthy control, $1=$ diabetes mellitus; Secretor $0=$ secretor, $1=$ Non-secretor)

\begin{tabular}{|l|c|c|c|}
\hline Secretor & Group 0 & Group 1 & Total \\
\hline 0 & 67 & 42 & 109 \\
& 33.50 & 21.21 & 27.39 \\
\hline 1 & 133 & 156 & 289 \\
& 66.50 & 78.79 & 72.61 \\
\hline Total & 200 & 198 & 398 \\
& 100.00 & 100.00 & 100.00 \\
\hline \multicolumn{4}{|l}{ Pearson Chi square $(1)=7.5545$} \\
\hline
\end{tabular}

Multivariate logistic regression analysis was performed between the healthy volunteer group and the group with diabetes mellitus. When adjusted for other (potential confounders) significant parameters in Univariate analysis viz., age, hypertension and blood group, secretors are nearly 2.1 times $(110 \%)$ at higher odds of having diabetes mellitus (odds ratio $=2.1$ ). When 
adjusted for WBC casts, age and hypertension, secretors are nearly 2.4 times at higher odds of having diabetes mellitus (odds ratio $=2.4$ ). In multivariate analysis, Blood group $\mathrm{B}$ was significantly associated with secretor status (p value $=<0.001)$. (Tables 7,8,9).

Table 7 Multivariate Logistic regression of secretors for age, diabetes, blood group and hypertension

\begin{tabular}{|c|c|c|c|c|c|c|}
\hline \multicolumn{3}{|c|}{ Logistic regression } & $\begin{array}{l}\text { nber of } \\
\mathrm{Chi}^{2}( \\
\mathrm{b}>\mathrm{Chi} \\
\text { udo } \mathrm{R}\end{array}$ & $\begin{array}{r}\mathrm{ss}=39 \\
=70.28 \\
0.0000 \\
0.150\end{array}$ & & \\
\hline Secretor & Odds Ratio & Std. Error & $\mathrm{Z}$ & $P>Z$ & $95 \%$ со & Interval \\
\hline $\mathrm{DM}$ & 2.130969 & 0.6836593 & 2.36 & 0.018 & 1.136303 & 3.996321 \\
\hline Age & 0.9941957 & 0.160869 & -0.36 & 0.719 & 0.9631606 & 1.026231 \\
\hline HT & 0.807016 & 0.0330899 & -6.14 & 0.000 & 0.361298 & 0.1802596 \\
\hline \multicolumn{7}{|c|}{ Blood Group } \\
\hline 1 & 0.9186675 & 0.2598521 & -0.30 & 0.764 & 0.527703 & 1.59929 \\
\hline 2 & 0.2902595 & 0.0994978 & -3.61 & 0.00 & 0.148253 & 0.5682889 \\
\hline 3 & 0.4113257 & 0.279514 & -1.31 & 0.191 & 0.108552 & 1.558597 \\
\hline
\end{tabular}

Table 8 Multivariate Logistic regression of diabetes for secretors, age and hypertension

\begin{tabular}{|c|c|c|c|c|c|c|}
\hline \multicolumn{3}{|c|}{ Logistic regression } & $\begin{array}{l}\text { ber of } \\
\text { Chi }{ }^{2}(6 \\
>\mathrm{Chi}^{2} \\
\text { Ido R2 }\end{array}$ & $\begin{array}{l}=398 \\
=345.52 \\
0000 \\
4450\end{array}$ & & \\
\hline DM & Odds Ratio & Std. Error & $\mathrm{Z}$ & $P>Z$ & $95 \%$ con & interval \\
\hline Secretor & 2.125107 & 0.6863043 & 2.33 & 0.020 & 1.128456 & 4.001996 \\
\hline Age & 1.105483 & 0.0221705 & 5.00 & 0.000 & 1.062873 & 1.149802 \\
\hline HT & 70.1241 & 31.82345 & 9.37 & 0.000 & 28.81232 & 170.6697 \\
\hline \multicolumn{7}{|c|}{ Blood Group } \\
\hline 1 & 0.9852747 & 0.3520296 & -0.04 & 0.967 & 0.4891374 & 1.984649 \\
\hline 2 & 1.313316 & 0.4828664 & 0.74 & 0.459 & 0.6388641 & 2.699792 \\
\hline 3 & 0.4672713 & 0.3841435 & -0.93 & 0.355 & 0.0932817 & 2.340677 \\
\hline
\end{tabular}

Table 9 Multivariate Logistic regression of secretors for diabetes, WBC casts, age and hypertension

\begin{tabular}{|c|c|c|c|c|c|c|}
\hline \multicolumn{3}{|c|}{ Logistic regression } & \multicolumn{4}{|c|}{$\begin{array}{l}\mathrm{LR} \mathrm{chi}^{2}(7) \quad=71.61 \\
\text { Prob }>\operatorname{chi}^{2}=0.0000\end{array}$} \\
\hline \multicolumn{3}{|c|}{ Log likelihood $=-197.84645$} & Pseudo R2 & $=0.1$ & & \\
\hline Secretor & Odds Ratio & Std. Error & $\mathrm{Z}$ & $P>Z$ & \multicolumn{2}{|c|}{$95 \%$ conf. interval } \\
\hline DM & 2.401449 & 0.8120429 & 2.59 & 0.010 & 1.237776 & 4.659128 \\
\hline WBC cast & 0.5572227 & 0.2888805 & -1.13 & 0.259 & 0.2017164 & 1.539276 \\
\hline Age & 0.9944477 & 0.0161011 & -0.34 & 0.731 & 0.9633857 & 1.026511 \\
\hline HT & 0.0814148 & 0.0334764 & -6.10 & 0.000 & 0.0363667 & 0.1822648 \\
\hline \multicolumn{7}{|c|}{ Blood Group } \\
\hline 1 & 0.9499435 & 0.2703407 & -0.18 & 0.857 & 0.5438231 & 1.65935 \\
\hline 2 & 0.2890725 & 0.0992846 & -3.61 & 0.000 & 0.1474531 & 0.5667084 \\
\hline 3 & 0.4150275 & 0.2831756 & -1.29 & 0.197 & 0.1089676 & 1.580725 \\
\hline
\end{tabular}

Pearson Chi Square test was performed within the study group (diabetes mellitus) for association of secretor status, duration of diabetes and various complications. Non-Secretor status is significantly associated culture positive urinary tract infection ( $\mathrm{p}$ value $=0.005)$. Secretor status is significantly associated with hypertension ( $\mathrm{p}$ value $<0.001$ ). WBC casts were significantly associated with study group when compared with healthy control group ( $\mathrm{p}$ value $<0.001$ ).

Two sample $t$ test with unequal variances was performed and the following parameters were found to be significant. Non-secretor status is significantly associated with increasing duration of diabetes ( $p$ value $=0.0176$ ). Increased HbA1c level is significantly associated with both 
microalbuminuria, macroalbuminuria, retinopathy and neuropathy ( $\mathrm{p}$ value $<0.001$ ).

Raise in HbA1c level is significantly associated with duration of diabetes ( $\mathrm{p}$ value $<0.001$, ANOVA, Barlett test for equal variances) and also with increase in serum creatinine ( $\mathrm{p}$ value $<0$ .001, ANOVA).

\section{Secretor status- Heamagglutination}

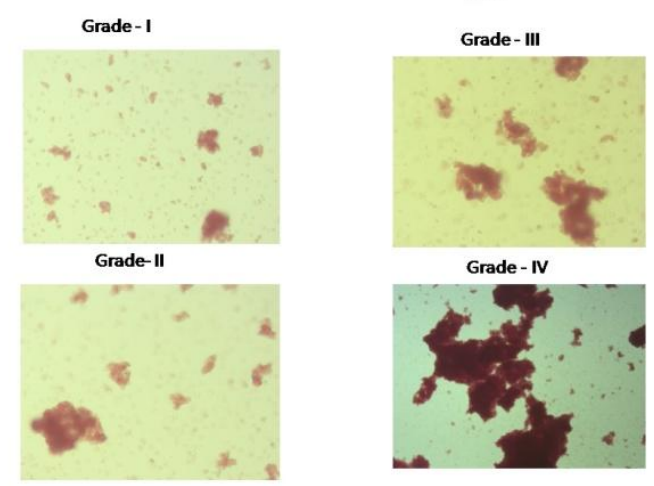

Fig 01

\section{Discussion}

Non-secretor status has been associated in the pathogenesis of diseases such as inflammatory bowel disease, rheumatic fever and increased predisposition to infections ${ }^{16}$.

Diabetic non-secretors appear to have lower levels of complement fractions when compared to diabetic secretors ${ }^{17}$. In our study, Non-secretors contributed to $66.5 \%$ in the healthy controls, while in patients with type II diabetes mellitus, $79 \%$ of the patients were non-secretors. Non-secretors constituted a much higher proportion in South Indian population when compared with Caucasian population $^{18}$, where only $20 \%$ of the population were non-secretors. Our study results differ from a similar study in Pakistan19, where around $35 \%$ of their healthy population were non-secretors. The difference is significant as the method used is by adsorption-inhibition method in all the studies with serial dilutions.

However, in a recent study from Bangladesh ${ }^{20}$, the non-secretor status ascertained by FUT2 genotyping status was $40 \%$, these variations may be due to significant genetic variations across various geographic regions, thus highlighting the need to analyze this factor in greater detail. (Table 10)

Table 10 Comparison of Secretor status in different populations

\begin{tabular}{|l|c|c|c|c|c|}
\hline S.No & $\begin{array}{c}\text { Non-secretors } \\
\text { inhealthy } \\
\text { controls in our } \\
\text { study }\end{array}$ & $\begin{array}{c}\text { Study from } \\
\text { Rajasthan } \\
\text { (Metgud R et al } \\
\text { 2016) [2] }\end{array}$ & $\begin{array}{c}\text { Caucasian } \\
\text { population } \\
\text { (McGovern DP et } \\
\text { al, 2010) [16] }\end{array}$ & $\begin{array}{c}\text { Study from } \\
\text { Karachi } \\
\text { (Saboor M et al } \\
\text { 2014) [19] }\end{array}$ & $\begin{array}{c}\text { Study from } \\
\text { Bangladesh } \\
\text { (Mottram L et } \\
\text { al 2017) [20] }\end{array}$ \\
\hline 1 & $66.5 \%$ & $20 \%$ & $20 \%$ & $35 \%$ & $40 \%$ \\
\hline
\end{tabular}

We also found significant increase in the percentage of non-secretors, constituting around $79 \%$ in the patients with type II diabetes mellitus. The increase in percentage of non-secretors in correlation with healthy controls was found to have strong statistical significance.

On Univariate analysis between the healthy control group and the diabetes group, age, hypertension and secretor status were significantly associated. Multivariate analysis was performed with logistic regression and when adjusted for age, hypertension and blood group, secretors are nearly 2.1 times $(110 \%)$ at higher odds of diabetes mellitus. WBC casts, age and hypertension were adjusted by logistic regression and secretors are nearly 2.4 times at higher odds of diabetes mellitus (odds ratio $=2.4$ ). Thus, though nonsecretors appear to be predisposed to diabetes mellitus, when the data is analysed in comparison with healthy control and adjusted for confounding factors such as age and hypertension, secretors are at higher odds of developing diabetes mellitus. Our data is similar to the study by Smyth et $\mathrm{al}^{21}$, who found significant association of non-secretors with type I diabetes mellitus in a study design comprising of both healthy controls and study subjects.

In our study, we also found significant association of non-secretor status with duration of diabetes mellitus and culture positive UTI, while clinically 
suspected and UTI with positive WBC casts in urine did not show association. HbAlc levels were significantly associated with microangiopathic complications of diabetes mellitus which is similar to several other studies in the literature.

Non-secretors were also common in the elderly. We could not find any report in the literature with this association. Possible heterozygous non-sense and frameshift mutations in FUT2 gene, which is a fetal gene involved in innate immunity may possibly be a reason for the same. However, the exact cause and effect association of age and nonsecretor status was not studied in this work. Another interesting novel finding in the study was the significant association of hypertension with secretor status ( $p$ value $<0.001$ ). We did not find any literature with the association of secretor status and hypertension. The possible indirect association that we hypothesize is that gut microbiota is significantly associated with both systolic and diastolic hypertension ${ }^{22}$, and at the same time secretor status is proven to have significant role in the modulation of gut microbiome ${ }^{12}$. However, the direct cause and effect association of hypertension with secretor status needs further study.

Association of type I diabetes has been reported with non-secretors earlier ${ }^{21}$. In our study too, if the analysis was confined only to the study group of patients with type II diabetes mellitus, nonsecretors had significant association with type II diabetes mellitus ( $p$ value $=0.006$ ). However, on Cox multivariate regression analysis, after eliminating the potential confounders age and hypertension, secretor status was significantly associated with type II diabetes mellitus with over $110 \%$ higher odds (Odds ratio - 2.1).

In our study, non-secretor status was significantly associated with culture positive urinary tract infections and this was similar to the results from various other studies ${ }^{23,24}$.All the patients with UTI included in our study group had lower urinary tract infection and we did not have any patients with pyelonephritis in our analysis.
Our results were however contradictory to the study by Smyth DJ et $\mathrm{al}^{21}$, who had described increased resistance to infection in association with non-secretors.

We did not find any significant association of secretors with microvascular complications of type II diabetes mellitus. Raise in HbA1c levels showed significant association with retinopathy, neuropathy and diabetic nephropathy with increase in microalbuminuria, macroalbuminuria as well as serum creatinine levels ( $\mathrm{p}$ value < 0.001). Our findings are similar to the findings from Ma et $\mathrm{al}^{25}$.

In our study, we did not carry out genotyping for various alleles of $\mathrm{HbA} 1 \mathrm{c}$ but however in one of the earlier studies, genome wide association studies of the HbA1c locus could not show any association with complications of type II diabetes mellitus in Asian population ${ }^{26}$.

Another interesting observation that is highlighted in our study on multivariate analysis is the significant association of blood group B with secretor status. We could not get any literature on this association in the same population. However, in a study from Iraq, blood group $\mathrm{O}$ was found to be significantly associated with secretors ${ }^{27}$.

The major limitation in the study is the crosssectional nature of the study with lack of followup. However, the findings observed are novel and merit further attention and analysis in a larger population.

Sources of support in the form of grants - Nil Institute Human Ethics Committee Approval number - No. GHIEC/2016 dated 22/12/2016)

\section{References}

1. Prakobphol A, Leffler H, Fisher SJ. The high molecular-weight human mucin is the primary salivary carrier of $\mathrm{ABH}, \mathrm{Le}(\mathrm{a})$, and Le(b) blood group antigens. Crit Rev Oral Biol Med 1993;4:325-333.

2. Metgud R, Khajuria N, Mamta GR. Evaluation of the Secretor Status of ABO Blood Group Antigens in Saliva among 
Southern Rajasthan Population Using Absorption Inhibition Method. Journal of clinical and diagnostic research 2016;10:ZC01-ZC03

3. Albertolle ME, Hassis ME, Ng CJ, Cuison S, Williams K, Prakobphol A, et al. Mass spectrometry based analyses showing the effects of secretor and blood group status on salivary N-glycosylation. Clinical Proteomics 2015;12:1-16

4. Patrick AW, Collier A: An infectious aetiology of insulin-dependent diabetes mellitus? Role of the secretor status. FEMS Microbiol Immunol 1989;1:411-416.

5. D'Adamo PJ, Kelly GS. Metabolic and immunologic consequences of $\mathrm{ABH}$ secretor and Lewis subtype status, Alternative Medicine Review 2001;6 (4):395.

6. Suadicani P, Hein HO, Gyntelberg F: Genetic and life-style determinants of peptic ulcer. A study of 3,387 men aged 54 to 74 years: The Copenhagen Male Study. Scand J Gastroenterol 1999;34:12-17.

7. Kulkarni DG, Venkatesh D: Non-secretor status; a predisposing factor for vaginal candidiasis. Indian J Physiol Pharmacol 2004;48:225-229.

8. Lomberg H, Jodal U, Leffler H, De Man P, Svanborg C. Blood group non-secretors have an increased inflammatory response to urinary tract infection. Scand J Infect Dis. 1992;24:77-83

9. Yazawa S, Yokobori T, Ueta G, Ide $M$, Altan B, Thongprachum A, et al. Blood group substances as potential therapeutic agents for the prevention and treatment of infection with Noroviruses proving novel binding patterns in human tissues. PLoS ONE 2014;9: e89071.

10. Nasir W, Frank M, Koppisetty CAK, Larson G, Nyholm PG. Lewis histo blood group $\alpha 1,3 / \alpha 1,4$ fucose residues both mediate binding to GII.4 noroviruses. Glycobiology 2012;22:1163-1172.
11. Trang NV, Vu TH, Le NT, Huang P, Jiang $\mathrm{X}$, Anh DD. Association between Norovirus and Rotavirus infection adnHisto-blood group antigen types in Vietnamese Children. Journal of Clinical Microbiology 2014;52:1366-1374.

12. Wacklin P, Tuimala J, Nikkila J, TIms S, Makivuokko H, Alakulppi N, et al. Fecal Microbiota composition in adults is associated with FUT2 gene determining the secretor status. PloS one 2014;9: e94863.

13. Abd ST. Individual variations in secretory and group antigen. International Journal of Science and Nature 2015;6:447-451.

14. Murray PR, Baron EJ, Pfaller MA, Tenover FC, Yolken RH. Manual of Clinical Microbiology. 6th Edition. Washington, DC: ASM Press; 1995

15. Clinical and Laboratory Standards Institute (CLSI) (2016) Performance standards for antimicrobial susceptibility testing; twentysixth informational supplement. CLSI document M100-S21. The Institute, Wayne (PA)

16. McGovern DP, Jones MR, Taylor KD, Marciante K, Yan X, Dubinsky M, et al. Fucosyltransferase 2 (FUT2) non-secretor status is associated with Crohn's disease. Human molecular genetics.2010 Jun 22; $19: 3468-76$

17. Blackwell CC, Weir DM, Patrick AW, et al. Secretor state and complement levels (C3 andC4) in insulin dependent diabetes mellitus. Diabetes Res 1988;9:117-119

18. Peters WH, Gohler W. ABH-secretion and Lewis red cell groups in diabetic and Normal subjects from Ethiopia. ExpClin Endocrinol 1986;88:64-70.

19. Saboor M, Ullah A, Qamar K, Mir A. Frequency of $\mathrm{ABH}$ secretors and non secretors: A cross sectional study in Karachi. Pakistan journal of medical sciences 2014;30:189.

20. Mottram L, Gudrun G, Larson G, Qadri F, Svennerholm AM. FUT2 non-secretor 
status is associated with altered susceptibility to symptomatic enterotoxigenic Escherichia coli infection in Bangladeshis. Scientific reports 2017;7: 10649.

21. Smyth DJ, Cooper JD, Howson JMM, Clarke P, Downes K, Mistry T, et al. FUT2 non secretor status links type I diabetes susceptibility and resistance to infection. Diabetes 2011;60:3081-3084.

22. Gomez-Arango LF, Barrett HL, Mcintyre D, Callaway LK, Morrison M, Nitert MD. Increased systolic and diastolic blood pressure is associated with altered gut microbiota composition and butyrate production in early pregnancy. Hypertension 2016;68:974-981.

23. Ishitoya $\mathrm{S}$, Yamamoto $\mathrm{S}$, Mitsumori $\mathrm{K}$, Ogawa O, Terai A. Non secretor status is associated with female acute uncomplicated pyelonephritis. British Journal of Urology International 2002;89:851-854.

24. Sheinfeld J, Schaeffer AJ, Cordon-Cardo C, Rogatko A, et al. Association of the Lewis blood-group phenotype with recurrent urinary tract infections in women. $\mathrm{N}$ Engl J Med 1989;320:773-777.

25. Ma. J, Yang W, Fang N, Zhu W, Wei M. The association between intensive glycemic control and vascular complications in type 2 diabetes mellitus: A meta-analysis. Nutrition, Metabolism and Cardiovascular diseases 2009;19:596-603.

26. Chen P, Ong RT-H, Tay W-T, Sim X, Ali $\mathrm{M}$, et al. A Study Assessing the association of Glycated Hemoglobin A1C (HbA1C) Associated Variants with HbA1C, Chronic Kidney Disease and Diabetic Retinopathy in Populations of Asian Ancestry. PLoS ONE 2013;8: e79767.

27. Jaff MS. Higher frequency of secretor phenotype in $\mathrm{O}$ blood group-its benefits in prevention and/or treatment of some diseases. International journal of nanomedicine. 2010;5:901. 